\title{
Evidence of Double-Walled AI-Ge Imogolite-Like Nanotubes. A Cryo-TEM and SAXS Investigation
}

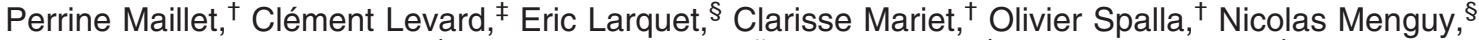 \\ Armand Masion, ${ }^{\ddagger}$ Emmanuel Doelsch," Jérôme Rose, ${ }^{\ddagger}$ and Antoine Thill ${ }^{\star, \dagger}$ \\ CEA, IRAMIS, Laboratoire Interdisciplinaire sur l'Organisation Nanométrique et Supramoléculaire 91191 \\ Gif-sur-Yvette, France, CEREGE, UMR 6635 CNRS/Aix Marseille Université, Europôle de l'Arbois, 13545 Aix en \\ Provence, France, Institut de Minéralogie et Physique des Milieux Condensés UMR 7590, CNRS, Université Pierre \\ et Marie Curie, Université Paris Diderot, Institut de Physique du Globe de Paris, 140 rue de Lourmel, 75015 Paris, \\ France, and CIRAD, UPR Recyclage et risque, F-34398 Montpellier, France
}

Received October 13, 2009; E-mail: antoine.thill@cea.fr

Since the discovery of carbon nanotubes (NTs), there has been great interest in the synthesis and characterization of similar shaped structures like inorganic nanotubes, nanorods, or nanowires. ${ }^{1}$ However, limitations such as purity, complexity of the protocol, low-yield production, and size polydispersity still remain major impediments for industrial-scale applications. In this context, synthetic imogolites appear as an exception. Imogolites are singlewalled aluminosilicate NTs of $2 \mathrm{~nm}$ diameter and up to $1 \mu \mathrm{m}$ in length with the empirical formula $(\mathrm{OH})_{3} \mathrm{Al}_{2} \mathrm{O}_{3} \mathrm{SiOH}^{2}$ whose structure has been determined using X-ray Diffraction (XRD), ${ }^{2}$ solid state Nuclear Magnetic Resonance (NMR), and Transmission Electron Microscopy (TEM). ${ }^{3}$ Imogolite analogues with the generic formula $(\mathrm{OH})_{3} \mathrm{X}_{2} \mathrm{O}_{3} \mathrm{YOH}$ (where $\mathrm{X}=\mathrm{Al}, \mathrm{Ga}$, or In and $\mathrm{Y}=\mathrm{C}$, $\mathrm{Si}, \mathrm{Ge}$, or $\mathrm{Sn}$ ) have been considered, mainly from a theoretical point of view, ${ }^{4}$ to tailor these NTs to specific needs. However, to date, only Ge imogolite analogues ${ }^{5}$ have been successfully synthesized. Although early reports of their synthesis involved diluted (i.e., millimolar) conditions, these imogolite analogues were recently obtained from 100 times more concentrated solutions, ${ }^{6}$ thereby opening the route for large scale applications. These analogues have been described to be structurally identical to the Al-Si imogolite, except for a larger tube diameter $(\sim 3.3 \mathrm{~nm})$ and shorter length (less than $100 \mathrm{~nm}$ ).

In the present study, the structure of two sets of samples obtained following the synthesis protocol described by Levard et al. ${ }^{6}$ with initial $\mathrm{Al}$ concentrations of 0.25 and $0.5 \mathrm{M}$ is obtained using different probes. Infrared spectroscopy (see Supporting Information (SI)) confirms that the local structure of the formed Al-Ge NTs is compatible with the one of imogolite. Their mesostructure has been examined by cryo-TEM and Small Angle X-ray Scattering (SAXS). We demonstrate here that $\mathrm{Al}-\mathrm{Ge}$ imogolite-like NTs synthesized at $0.25 \mathrm{M}$ are double-walled NTs with a low dispersion in diameter. SAXS data also suggest that the double-walled NTs consist of two concentric tubes of equal length and identical wall structure. At higher concentration $(0.5 \mathrm{M})$, both SAXS and cryo-TEM data confirm the formation of single-walled NTs.

A high resolution TEM observation of all types of imogolite is very difficult because of their fragility under the electron beam and the low electronic density contrast of the NTs. ${ }^{5 b}$ Therefore, in this study, we have used a cryo-TEM microscope operated using the minimum dose system (maximum electron flux 10 electrons per $\AA^{2}$ per s). The solutions were quick frozen in liquid ethane and

\footnotetext{
${ }^{\dagger}$ CEA.

* CEREGE.

Université Paris Diderot.

"CIRAD.
}

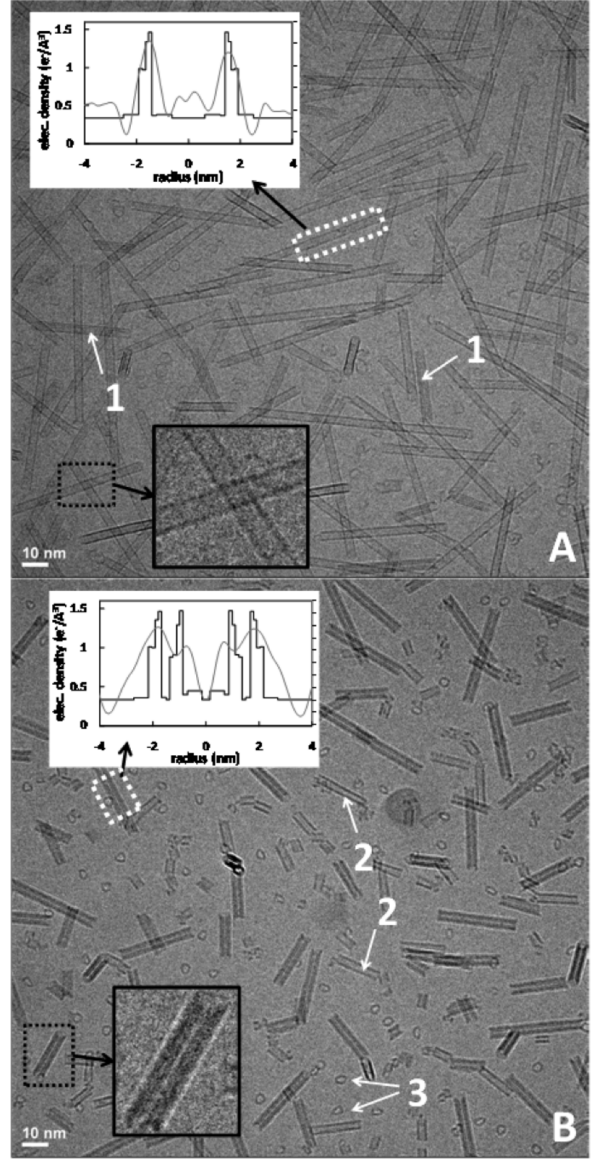

Figure 1. Cryo-TEM micrographs of $0.5 \mathrm{M}(\mathrm{A})$ and $0.25 \mathrm{M}$ (B) $\mathrm{Al}-\mathrm{Ge}$ imogolite-like NTs. Figures show the presence of two populations (1: double-walled tube and 2: single-walled tube) and the coexistence of the final tubes with synthesis precursors (3). Gray scale profiles averaged over the length of a single tube (white rectangle) are shown in arbitrary units (inset). Electron density profiles $\left(\mathrm{e}^{-} / \mathrm{A}^{3}\right)$ used for SAXS calculations are also reported.

observed at a temperature of $-180{ }^{\circ} \mathrm{C}$ in a JEOL $2100 \mathrm{~F}$ electron microscope using an accelerating voltage of $200 \mathrm{kV}$. In slight underfocus conditions (100 to $200 \mathrm{~nm}$ ), it has been possible to collect contrasted images up to a magnification of 200000 without significant sample structure modifications (see SI). Figure 1 shows the high resolution images of the NTs synthesized with an aluminum initial concentration of $0.5 \mathrm{M}$ (image $\mathrm{A}$ ) and $0.25 \mathrm{M}$ (image $\mathrm{B}$ ). 


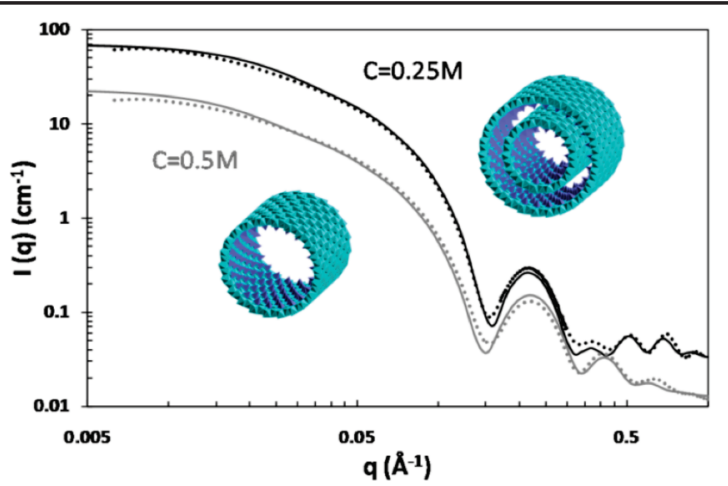

Figure 2. SAXS curves of 0.5 and $0.25 \mathrm{M} \mathrm{Al}-\mathrm{Ge}$ imogolite-like NTs. Experimental data are plotted using dots, and theoretical scattering curve of the represented structure are plotted using straight line. The $0.25 \mathrm{M}$ curve is plotted using a $\times 4$ offset.

At $0.5 \mathrm{M}$, the gray scale analysis, which is proportional to the electronic density, of sections taken perpendicular to the tube axis (see insert Figure 1A) shows a single maximum for the majority (99\%) of the tubes. This is consistent with a single-walled structure. On the contrary, the same analysis for the tubes obtained at 0.25 M clearly shows two maxima revealing that most of the tubes (96\%) are double-walled. To the best of our knowledge this is the first evidence of any double-walled imogolite-like NTs. Furthermore, the NTs are shorter than in the case of the $0.5 \mathrm{M}$ synthesis. In both samples, the tubes coexist with small particles of $\sim 5 \mathrm{~nm}$ in size. These particles very likely correspond to the structurally ill-defined precursors from which the NTs form ${ }^{3 \mathrm{~b}, 5 \mathrm{~b}}$ and for which a generic structure in the form of roof tile shaped fragments has been recently proposed. $^{7}$

SAXS measurements gave further quantitative insight into the structure of the NTs. The SAXS analysis on solutions has been performed on the BM02 beamline at the European Synchrotron Radiation Facility (ESRF, Grenoble, France). As expected, the two samples yield two different intensity profiles as shown on Figure 2. The single-walled NTs synthesized at $0.5 \mathrm{M}$ show regular oscillations which are well simulated with a model of single-walled $\mathrm{Al}-\mathrm{Ge}$ imogolite with a low dispersion in the tube diameter. The radial electronic density profile (source of the interaction with the $\mathrm{X}$-rays in the SAXS experiments) (Figure 1) has been computed from $\mathrm{Al}-\mathrm{Ge}$ imogolite models with a varying number of $\mathrm{Ge}$ atoms in the circumference. Despite the defocused cryo-TEM images which alter the absolute distances, the experimental gray scale cross sections compare reasonably well with the electronic density profiles (Figure 1). The best agreement with SAXS data is obtained using an average diameter of $3.5 \pm 0.15 \mathrm{~nm}$. A slightly elliptical ${ }^{8}$ rather than cylindrical cross section of the imogolite could explain the small mismatch in the minima positions between the calculated and experimental intensities.

The intensity of the $0.25 \mathrm{M}$ sample displays unexpected modulations at a large scattering vector revealing an additional structuration at smaller distances. Indeed, these modulations could be simulated using double-walled NT models with a low dispersion in tube diameter. The electronic density of the two walls was computed using the same atomic structure (Figure 2). Again, the agreement between the computed electronic density profile and the gray scale section of the cryo-TEM images is good (Figure 1). The diameter $(4.0 \pm 0.1 \mathrm{~nm})$ of the external double-walled NTs is larger than the single-walled NTs. The internal NT is smaller with a diameter of $2.4 \pm 0.1 \mathrm{~nm}$. The distance between the Ge layer of the external tube and the Al layer of internal tube is $2.7 \AA$ to reproduce the intensity modulations. Modifications of this distance between the two tubes in the SAXS model significantly change the intensity modulations at a large scattering vector (see SI). Thus the $2.7 \AA$ distance between the two NTs is precisely known and corresponds to the typical value of one water layer. This distance prevents any covalent links between the two tubes. A third remarkable requirement is that the inner and outer tubes must have the same length to reproduce the scattering data (see SI). SAXS results are not sensitive to the precise scattering length densities. A decrease of the $\mathrm{Al}$ polymerization level at higher concentration which may be the source of the different structure would not be detected. However, it is strongly dependent and precise in the determination of the radius and single or double wall structure.

The experimental observation of almost discrete curvatures for the Al-Ge imogolite-like NTs is not consistent with simulations showing that the total internal energy (potential and kinetic) of $\mathrm{Al}-\mathrm{Ge}$ imogolite NTs is not very sensitive to the number of $\mathrm{Al}$ atoms in the circumference. ${ }^{9}$ An additional factor needs to be identified to account for the observed low dispersion in the tube diameters.

Considering the research efforts targeted on carbon double- or multiwall NTs, the present identification of a simple route to obtain inorganic double- (multi-) walled structures is likely to trigger renewed interest in these materials and associated potential applications (e.g., gas storage under difficult temperature or pressure conditions), especially since they are known for their enhanced thermal and mechanical resistance properties with respect to the corresponding single-walled product.

Acknowledgment. We are grateful to Isabelle Morfin and Françoise Bley for their help during the SAXS experiment at ESRF beamline BM2 and to program C'Nano Ile de France for supporting cryo-electron microscopy at IMPMC. Laurent Michot and Manuel Pelletier are acknowledged for their help with IR spectroscopy.

Supporting Information Available: IR characterization, analysis of the electron beam damage and effect of double-walled gap width, the polydispersity, and the comparative length of inner and outer tube on SAXS curves are illustrated. This material is available free of charge via the Internet at http://pubs.acs.org.

\section{References}

(1) (a) Mayya, K. S.; Gittins, D. I.; Dibaj, A. M.; Caruso, F. Nano Lett. 2001, 1 (12), 727-730. (b) Wang, C. C.; Kei, C. C.; Yu, Y. W.; Perng, T. P. Nano Lett. 2007, 7, 1566-1569. (c) Lisiecki, I.; Sack-Kongehl, H.; Weiss, K.; Urban, J.; Pileni, M. P. Langmuir 2000, 16 (23), 8802-8806. (d) Liu, Z. W. Bando, Y. Adv. Mater. 2003, 15 (4), 303-305. (e) Zhao, A. W.; Meng, G. W.; Zhang, L. D.; Gao, T.; Sun, S. H.; Pang, Y. T. Appl. Phys. A 2003, 76 (4), 537-539.

(2) Cradwick, P. D. G.; Farmer, V. C.; Russel, J. D.; Masson, C. R.; Wada, K.; Yoshinaga, N. Nat. Phys. Sci. 1972, (240), 187-189.

(3) (a) Wada, K.; Yoshinaga, N. Am. Mineral. 1969, (54), 50-71. (b) Yang, H.; Wang, C.; Su, Z. Chem. Mater. 2008, (20), 4484-4488.

(4) Alvarez-Ramirez, F. J. Comput. Theor. Nanosci. 2009, (6), 1120-1124.

(5) (a) Wada, S.; Wada, K. Clays Clay Miner. 1982, (30), 123-128. (b) Mukherjee, S.; Kim, K.; Nair, S. J. Am. Chem. Soc. 2007, (129), 68206826.

(6) Levard, C.; Rose, J.; Masion, A.; Doelsch, E.; Borschneck, D.; Olivi, L.; Dominici, C.; Grauby, O.; Woicik, J. C.; Bottero, J.-Y. J. Am. Chem. Soc. 2008, 130 (18), 5862-5863.

(7) Levard C.; Rose J.; Thill A.; Masion A.; Doelsch E.; Maillet P.; Spalla O.; Olivi L.; Cognigni A.; Ziarelli F.; Bottero J.-Y. Chem. Mater. 2009, submitted

(8) Creton, B.; Bougeard, D.; Smirnov, K. S.; Guilment, J.; Poncelet, O. J. Phys. Chem. 2008, 112, 10013-10020.

(9) Konduri, S.; Mukherjee, S.; Nair, S. ACS Nano 2007, (1), 393-402.

JA908707A 